\title{
Dialister succinatiphilus sp. nov. and Barnesiella intestinihominis sp. nov., isolated from human faeces
}

Correspondence

Masami Morotomi

masami-morotomi@yakult.co.jp

\author{
Masami Morotomi, Fumiko Nagai, Hiroshi Sakon and Ryuichiro Tanaka \\ Yakult Central Institute for Microbiological Research, 1796 Yaho, Kunitachi, 186-8650 Tokyo, \\ Japan
}

Two anaerobic, non-spore-forming, bacteria (YIT $11850^{\top}$ and YIT $11860^{\top}$ ) that stained Gramnegative, were isolated from human faeces. Cells of strain YIT $11850^{\top}$ were coccobacilli, asaccharolytic and largely unreactive, with only traces of lactate and propionate as metabolic end products; however, strain YIT $11850^{\top}$ was able to decarboxylate succinate to propionate. The DNA G $+C$ content of strain YIT $11850^{\top}$ was 51.9 mol\%. Following $16 \mathrm{~S}$ rRNA gene sequence analysis, this strain was found to be most closely related to Dialister propionicifaciens, with $95.1 \%$ sequence similarity between the two taxa. Biochemical data supported the affiliation of strain YIT $11850^{\top}$ to the genus Dialister. Strain YIT $11850^{\top}$ therefore represents a novel species for which the name Dialister succinatiphilus sp. nov. is proposed; the type strain is YIT $11850^{\top}$ $\left(=\mathrm{DSM} 21274^{\top}=\mathrm{JCM} 15077^{\top}\right)$. Cells of the other isolate, strain YIT $11860^{\top}$, were non-motile, rod-shaped, positive for aesculin hydrolysis, negative for indole production, produced succinic and acetic acids as end products of glucose metabolism and possessed a DNA $G+C$ content of $45.5 \mathrm{~mol} \%$. On the basis of $16 \mathrm{~S}$ rRNA gene sequence similarity values, this strain was shown to belong to the family 'Porphyromonadaceae' related to Barnesiella viscericola ( $96.0 \%)$; similarity values with species within the family 'Porphyromonadaceae' with validly published names were less than $86 \%$. Biochemical data supported the affiliation of strain YIT $11860^{\top}$ to the genus Barnesiella. Strain YIT $11860^{\top}$ therefore represents a novel species for which the name Barnesiella intestinihominis sp. nov. is proposed; the type strain is YIT $11860^{\top}$ (=DSM $21032^{\top}=$ JCM $15079^{\top}$ ).
Many novel bacteria have been detected in the human gastrointestinal (GI) tract and faeces using cultureindependent approaches based on small subunit (16S and 18S) rRNA diversity (for review see Rajilić-Stojanović et al., 2007). The recent study of Eckburg et al. (2005) indicated that the proportion of reported cultivable bacteria was only $20 \%$ for faecal and mucosal samples of three healthy individuals. To better understand the physiological characteristics and function of the majority of human GI microbiota, several intensive cultivation trials aimed at isolating so-called 'unculturable' or 'as-yet-uncultured' bacteria from the human GI tract have been performed (Sakon et al., 2008). In this article, the isolation of two novel species from human faeces is reported. Although

Abbreviation: Gl, gastrointestinal.

The GenBank/EMBL/DDBJ accession numbers for the 16S rRNA gene sequences of strains YIT $11850^{\top}$ and YIT $11860^{\top}$ are AB370249 and AB370251, respectively.

$16 \mathrm{~S}$ rRNA gene sequence similarity values between Dialister succinatiphilus sp. nov. and other members of the genus Dialister are available with the online version of this paper. novel taxonomic units (species) based on a single isolate are proposed, these isolates displayed $>98 \%$ 16S rRNA gene sequence similarity to some of the human intestinal uncultured clones reported by several groups in the USA and other countries, as described below, indicating that these bacteria are common members of the human intestinal microbiota.

Faecal samples were collected from two healthy Japanese males (subjects C and M, 38 and 29 years old, respectively) and immediately transferred anaerobically. Each sample was weighed and diluted with pre-reduced $0.1 \mathrm{M}$ PBS (0.145 M NaCl, 0.15 M sodium phosphate; $\mathrm{pH} 7)$ in an anaerobic glove box (Coy Laboratory Products) containing $88 \%$ nitrogen, $7 \%$ hydrogen and $5 \%$ carbon dioxide. Then, each dilution was spread on modified Gifu anaerobic medium (GAM) agar (Nissui Pharmaceutical) and anaerobe basal agar (Oxoid). The composition of the modified GAM agar has been described in our previous report (Sakon et al., 2008). Plates were incubated at $37{ }^{\circ} \mathrm{C}$ for 3 days in the same anaerobic cabinet. Strain YIT $11850^{\mathrm{T}}$ was isolated from the GAM agar plate inoculated with a $10^{-8}$ serially diluted faecal sample from subject C. Strain 
YIT $11860^{\mathrm{T}}$ was isolated from an anaerobe basal agar plate inoculated with a $10^{-6}$ serially diluted faecal sample from subject M. Single colonies were picked and streaked out until single cultures were obtained on modified GAM agar. The end products of bacterial metabolism of glucose, lactate or succinate in pre-reduced peptone-yeast extract (PY) medium (Holdeman et al., 1977) supplemented with glucose (PYG medium), lactate or succinate, respectively, were analysed by HPLC according to a previously described procedure (Chonan et al., 1995). Cellular morphology was recorded after Gram-staining of 3 day plate or 1 day broth cultures. Biochemical characteristics were determined using the API Rapid ID 32A, API ZYM and API 20A systems (bioMérieux) according to the manufacturer's instructions. The DNA G $+\mathrm{C}$ content was determined by hydrolysing the DNA enzymically and quantifying the nucleosides by HPLC according to the method of Ezaki et al. (1990). Closely related sequences were retrieved from the DDBJ (DNA DataBank of Japan) using the FASTA program (Lipman \& Pearson, 1985). Sequences were aligned and used to produce an unrooted phylogenetic tree by the neighbour-joining method (Saitou \& Nei, 1987) using CLUSTAL_X (version 1.83) (Thompson et al., 1997). The stability of the groupings was estimated by bootstrap analysis (1000 replications) in CLUSTAL_X. Trees were visualized by using the TREEVIEW program (version 1.6.6) (Page, 1996). The minimal evolution method (1000 bootstrap replicates) in MEGA4 (Tamura et al., 2007) and the maximum-likelihood method from the PHYLIP program package (Felsenstein, 1993) were used to confirm the phylogenetic placement of the aligned sequences.

Cells of YIT $11850^{\mathrm{T}}$ stained Gram-negative and were obligately anaerobic, non-motile coccobacilli (0.4-
$0.9 \times 0.8-2.0 \mu \mathrm{m}$ ), occurring singly, in pairs and in short chains. Colonies after 3 days anaerobic incubation on ATCC medium 1257 (ETSA medium) were translucent, entire, circular, convex and pin-point. They were asaccharolytic in API test systems. Although the growth of YIT $11850^{\mathrm{T}}$ in PYG broth produced no visible turbidity, trace amounts of lactate and propionate were detected as end products of metabolism. In the API Rapid ID 32A and API ZYM test systems, strain YIT $11850^{\mathrm{T}}$ was very unreactive; apart from activities for esterase C4, naphthol-AS-BIphosphohydrolase, acid phosphatase and weak alkaline phosphatase, all other tests were negative. Addition of succinate, but not lactate, enhanced the growth of YIT $11850^{\mathrm{T}}$ and subsequent HPLC analysis revealed that this strain produced a large amount of propionate from PY medium supplemented with succinate. Strain YIT $11850^{\mathrm{T}}$ was able to decarboxylate succinate to propionate, similar to Dialister propionicifaciens (Jumas-Bilak et al., 2005) and members of the genus Veillonella. Enzymic profiles obtained with Rapid ID 32A highlighted the phenotypic differences within the genus Dialister, which are summarized in Table 1.

Approximately $1500 \mathrm{bp}$ of the 16S rRNA gene of strain YIT $11850^{\mathrm{T}}$ was sequenced and database searches revealed highest sequence relatedness to that of the type strain of $D$. propionicifaciens ( $95.1 \%$ sequence similarity; see supplementary Table S1 in IJSEM Online). Phylogenetic analysis of these and other related sequences was performed and data confirmed that strain YIT $11850^{\mathrm{T}}$ was phylogenetically most closely associated with $D$. propionicifaciens and Dialister invisus (Fig. 1). Currently, only four species of the genus Dialister are known: Dialister pneumosintes, formerly classified as Bacteroides pneumosintes and origin-

Table 1. Major characteristics of strain YIT $11850^{\top}$ and phylogenetically related species of the genus Dialister

Taxa: 1, strain YIT $11850^{\mathrm{T}}$; 2, Dialister propionicifaciens CCUG $49291^{\mathrm{T}}$; 3 , Dialister invisus CCUG $47026^{\mathrm{T}}$; 4, Dialister micraerophilus CCUG $48837^{\mathrm{T}}$; 5, Dialister pneumosintes ATCC $33048^{\mathrm{T}}$. Data for strain YIT $11850^{\mathrm{T}}$ are from this study. Data for other species are from Jumas-Bilak et al. (2005). Rapid ID 32A API codes for these type strains are 0000400000, 0000000000, 0000000000, 2000013305 and 0000012401, respectively. +, Positive; -, negative; NA, no data available.

\begin{tabular}{|c|c|c|c|c|c|}
\hline Characteristic & 1 & 2 & 3 & 4 & 5 \\
\hline Isolation source & Human faeces & $\begin{array}{c}\text { Human clinical } \\
\text { samples }\end{array}$ & $\begin{array}{c}\text { Human oral } \\
\text { cavity }\end{array}$ & $\begin{array}{c}\text { Human clinical } \\
\text { samples }\end{array}$ & $\begin{array}{l}\text { Human nasopharyn- } \\
\text { geal secretions }\end{array}$ \\
\hline \multicolumn{6}{|l|}{ Rapid ID 32A API: } \\
\hline Alanine arylamidase & - & - & - & + & - \\
\hline Alkaline phosphatase & + & - & - & - & - \\
\hline Arginine dihydrolase & - & - & - & + & - \\
\hline Glycine arylamidase & - & - & - & - & + \\
\hline Histidine arylamidase & - & - & - & + & + \\
\hline Leucine arylamidase & - & - & - & + & + \\
\hline Phenylalanine arylamidase & - & - & - & + & - \\
\hline Serine arylamidase & - & - & - & + & - \\
\hline
\end{tabular}




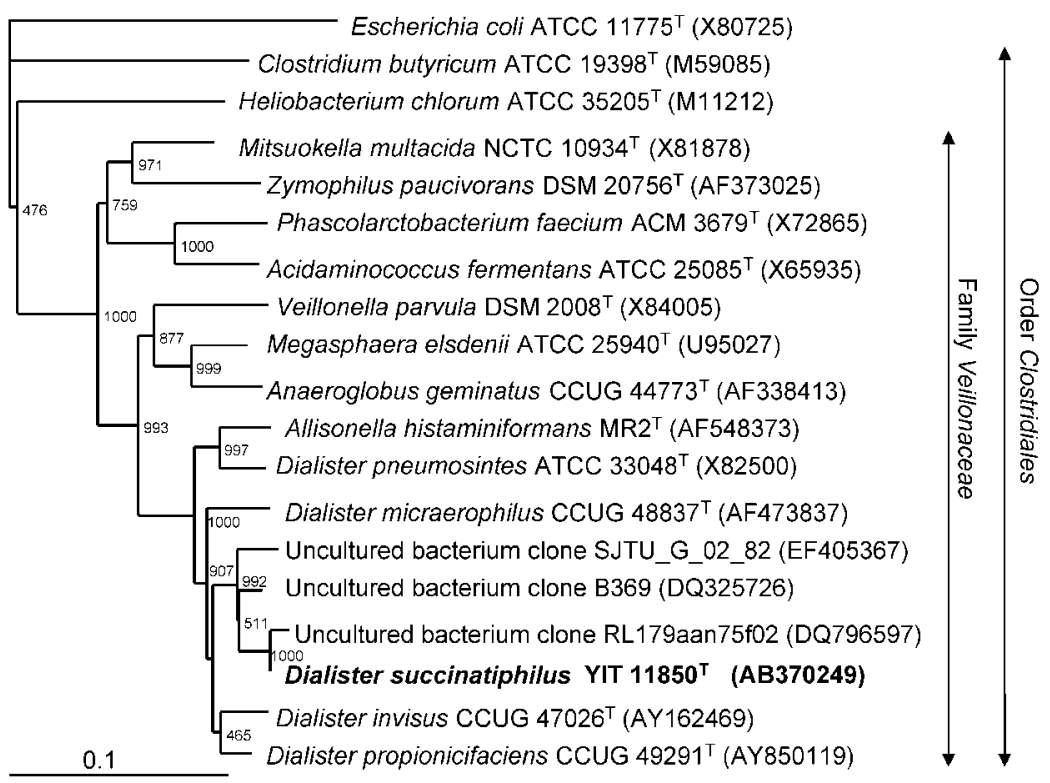

Fig. 1. Phylogenetic tree showing the positions of Dialister succinatiphilus YIT $11850^{\top}$ and other species belonging to the family Veillonaceae based on 16S rRNA gene sequence similarity data. The tree was rooted with Escherichia coli and constructed by using the neighbour-joining method; bootstrap values from 1000 replications are given at the nodes. GenBank/EMBL/DDBJ accession numbers are shown in parentheses. Bar, 0.1 substitutions per nucleotide position.

ally isolated from nasopharyngeal secretions of patients with influenza (Willems \& Collins, 1995); D. invisus, isolated from the human oral cavity (Downes et al., 2003); and Dialister micraerophilus and D. propionicifaciens, isolated from human clinical samples (Jumas-Bilak et al., 2005). Although there is no evidence that species of the genus Dialister have been isolated from human faeces as common members of human indigenous microbiota, many uncultured human intestinal bacterial clones with $16 \mathrm{~S}$ rRNA gene sequences that are highly similar to that of strain YIT $11850^{\mathrm{T}}$ have been deposited in the DDBJ (Ley et al., 2006; Gill et al., 2006) (Fig. 1). The DNA G + C content of strain YIT $11850^{\mathrm{T}}$ was $51.9 \mathrm{~mol} \%$, whereas that of the recognized species of the genus Dialister is $35-46 \mathrm{~mol} \%$ (Downes et al., 2003). It is evident from the results of the taxonomic study that the Gram-negative staining, coccobacillus strain YIT $11850^{\mathrm{T}}$ recovered from human faeces represents a hitherto unknown species.

Cells of YIT $11860^{\mathrm{T}}$ stained Gram-negative and were obligately anaerobic, non-motile, pleomorphic rods (0.4$1.0 \times 1.3-13.0 \mu \mathrm{m})$ that hydrolysed aesculin but were negative for indole production. Colonies after 3 days anaerobic incubation on anaerobe basal agar were 1$3 \mathrm{~mm}$ in diameter, translucent to pale orange-yellow in colour with irregular margins and a low convex shape. Analysis of metabolic end products by HPLC from PYG broth revealed succinic and acetic acids. Results based on the API Rapid ID 32A, 20A and API ZYM systems are summarized in Table 2. Many of the characteristics were similar to those of Barnesiella viscericola (Sakamoto et al., 2007).

An almost-complete 16S rRNA gene sequence of strain YIT $11860^{\mathrm{T}}$ was determined (1484 bp). The most similar $16 \mathrm{~S}$ rRNA gene sequences $(98.6-99.9 \%$ similarity) were derived from studies of uncultured colonic bacteria (Ley et al., 2006; Eckburg et al., 2005; Mai et al., 2006) (Fig. 2). The cultured bacterium most closely related to strain YIT $11860^{\mathrm{T}}$ was B. viscericola ( $96.0 \%$ similarity). B. viscericola, isolated from chicken caecum, is currently the sole recognized species of the genus Barnesiella. A tree showing the phylogenetic relationships of the unknown bacterium is depicted in Fig. 2 and reveals that strain YIT $11860^{\mathrm{T}}$ is related to B. viscericola. The DNA G $+\mathrm{C}$ content of strain YIT $11860^{\mathrm{T}}$ was $45.5 \mathrm{~mol} \%$.

In this report, the isolation of two novel species from human faeces is described. Their phenotypic criteria corresponded to earlier studies of the genera Dialister and Barnesiella, respectively. Based on their phylogenetic distinctiveness, it is considered that strain YIT $11850^{\mathrm{T}}$ represents a novel species in the genus Dialister and, on the basis of the presented findings, it is proposed that the unknown species from human faeces be assigned as a novel

Table 2. Major characteristics that differentiate strain YIT $11860^{\top}$ from the phylogenetically related bacterium Barnesiella viscericola

Data for YIT $11860^{\mathrm{T}}$ are from this study. Data for B. viscericola JCM $13660^{\mathrm{T}}$ are from Sakamoto et al. (2007).

\begin{tabular}{|lcc|}
\hline Characteristic & YIT 11860 $^{\mathbf{T}}$ & B. viscericola \\
\hline Isolation source & Human faeces & Chicken caecum \\
DNA G+C content (mol\%) & 45.5 & 52.0 \\
Acid production from: & & - \\
$\quad$ Lactose & + & + \\
Sucrose & - & - \\
Rapid ID 32A API: & & - \\
$\quad \beta$-Glucuronidase & + & \\
Fermentation of mannose & + & \\
\hline
\end{tabular}




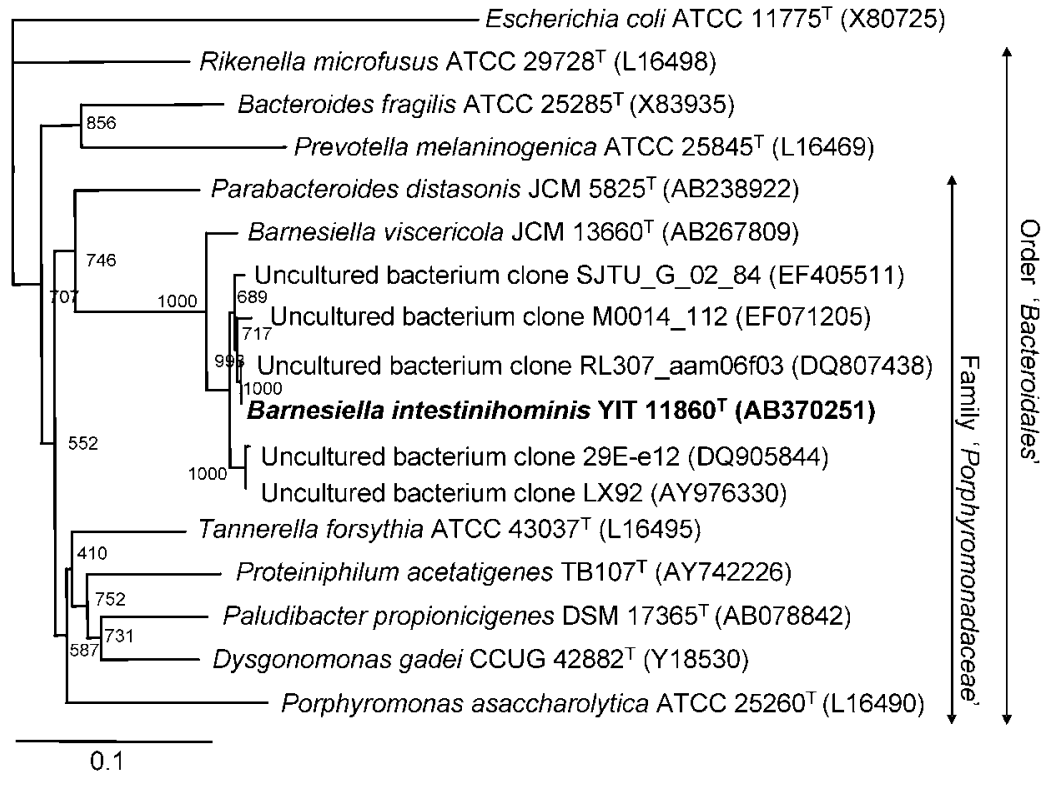

Fig. 2. Phylogenetic tree showing the position of strain YIT $11860^{\top}$ among selected clones or strains belonging to the family Porphyromonadaceae. The tree was rooted with Escherichia coli and constructed by using the neighbour-joining method; bootstrap values from 1000 replications are given at the nodes. GenBank/EMBL/DDBJ accession numbers are shown in parentheses. Bar, 0.1 substitutions per nucleotide position. species, Dialister succinatiphilus sp. nov. Likewise, on the basis of the presented findings, it is proposed that strain YIT $11860^{\mathrm{T}}$ be assigned to the genus Barnesiella as a novel species, Barnesiella intestinihominis sp. nov.

\section{Emended description of the genus Dialister (ex Bergey et al. 1923) Moore and Moore 1994}

The description is as emended by Downes et al. (2003) and Jumas-Bilak et al. (2005) with the following modification: the DNA G + C content ranges from 35 to $52 \mathrm{~mol} \%$.

\section{Description of Dialister succinatiphilus sp. nov.}

Dialister succinatiphilus (suc.ci.na.ti.phi'lus. N.L. n. succinas -atis succinate; Gr. adj. philos loving; N.L. masc. adj. succinatiphilus succinate-loving).

Cells are non-motile, non-spore-forming cocci to coccobacilli, approximately $0.4-0.9 \times 0.8-2.0 \mu \mathrm{m}$, which stain Gram-negative. Colonies after 3 days anaerobic incubation on ATCC medium 1257 (ETSA medium) are translucent, entire, circular, convex and pin-point. Oxidase- and catalase-negative. Aesculin and gelatin are not hydrolysed and nitrate is not reduced. Indole is not produced. Asaccharolytic. Esterase C4, naphthol-AS-BI-phosphohydrolase, acid phosphatase and weak alkaline phosphatase activities may be detected. Using the commercially available API test systems, no activity is detected for alanine arylamidase, $\alpha$-arabinosidase, arginine arylamidase, arginine dihydrolase, $\alpha$-chymotrypsin, cystine arylamidase, esterase lipase C8, $\alpha$-fucosidase, $\alpha$-glucosidase, $\beta$-glucosidase, $\beta$-glucuronidase, $\alpha$-galactosidase, $\beta$-galactosidase, 6 phospho- $\beta$-galactosidase, glutamic acid decarboxylase, glutamyl glutamic acid arylamidase, glycine arylamidase, histidine arylamidase, leucine arylamidase, leucyl glycine arylamidase, lipase $\mathrm{C} 4, \alpha$-mannosidase, $N$-acetyl- $\beta$-gluco- saminidase, proline arylamidase, phenylalanine arylamidase, phosphoamidase, pyroglutamic acid arylamidase, serine arylamidase, trypsin, valine arylamidase, urease or tyrosine arylamidase.

The type strain, YIT $11850^{\mathrm{T}} \quad\left(=\mathrm{DSM} \quad 21274^{\mathrm{T}}=\mathrm{JCM}\right.$ $\left.15077^{\mathrm{T}}\right)$, was isolated from human faeces. The DNA $\mathrm{G}+\mathrm{C}$ content of the type strain is $51.9 \mathrm{~mol} \%$.

\section{Emended description of the genus Barnesiella Sakamoto et al. 2007}

The description is as given by Sakamoto et al. (2007) with the following modification: the DNA $\mathrm{G}+\mathrm{C}$ content is in the range $45-52 \mathrm{~mol} \%$.

\section{Description of Barnesiella intestinihominis sp. nov.}

Barnesiella intestinihominis (in.tes.ti.ni.ho' mi.nis. L. gen. n. intestini of the intestine; L. gen. n. hominis of a human being; N.L. gen. n. intestinihominis of the human intestine).

Cells stain Gram-negative and are obligately anaerobic, non-motile, pleomorphic rods $(0.4-1.0 \times 1.3-13.0 \mu \mathrm{m})$ that hydrolyse aesculin, but are negative for indole production. Colonies after 3 days anaerobic incubation on anaerobe basal agar are $1-3 \mathrm{~mm}$ in diameter, translucent with irregular margins, pale orange-yellow in colour and low convex. Analysis of metabolic end products by HPLC from PYG broth reveals succinic and acetic acids. Catalase, urease and oxidase are not produced. Negative for nitrate reduction and gelatin hydrolysis. Acid is produced from glucose, lactose, maltose and D-mannose, but not from D-mannitol, sucrose, salicin, D-xylose, L-arabinose, glycerol, melezitose, raffinose, D-sorbitol, L-rhamnose or trehalose. Weak acid is produced from cellobiose. Using 
the commercially available API test systems, activity is detected for $N$-acetyl- $\beta$-glucosaminidase, acid phosphatase, alanine arylamidase, alkaline phosphatase, $\alpha$-galactosidase, $\beta$-galactosidase, $\alpha$-glucosidase, $\beta$-glucosidase, $\beta$-glucuronidase, glutamic acid decarboxylase, leucyl glycine arylamidase and naphthol-AS-BI-phosphohydrolase, but not for $\alpha$ arabinosidase, arginine arylamidase, arginine dihydrolase, chymotrypsin, cystine arylamidase, $\alpha$-fucosidase, 6 -phospho- $\beta$-galactosidase, glutamyl glutamic acid arylamidase, glycine arylamidase, histidine arylamidase, leucine arylamidase, lipase C4, $\alpha$-mannosidase, phenylalanine arylamidase, proline arylamidase, pyroglutamic acid arylamidase, serine arylamidase, trypsin, tyrosine arylamidase and valine arylamidase. Weak activity is detected for esterase lipase C8 and esterase C4.

The type strain, YIT $11860^{\mathrm{T}} \quad\left(=\mathrm{DSM} \quad 21032^{\mathrm{T}}=\mathrm{JCM}\right.$ $\left.15079^{\mathrm{T}}\right)$, was isolated from human faeces. The DNA $\mathrm{G}+\mathrm{C}$ content of the type strain is $45.5 \mathrm{~mol} \%$.

\section{Acknowledgements}

We are grateful to Professor Jean P. Euzéby of the Ecole Nationale Veterinaire in Toulouse for his suggestions regarding the etymology of the species epithet. We thank Dr Haruji Sawada for his understanding and encouragement through our research activities.

\section{References}

Bergey, D. H., Harrison, F. C., Breed, R. S., Hammer, B. W. \& Huntoon, F. M. (1923). In Bergey's Manual of Determinative Bacteriology, pp. 271-272. Baltimore: Williams \& Wilkins.

Chonan, O., Matsumoto, K. \& Watanuki, M. (1995). Effect of galactooligosaccharides on calcium absorption and preventing bone loss in ovariectomized rats. Biosci Biotechnol Biochem 59, 236-239.

Downes, J., Munson, M. \& Wade, W. G. (2003). Dialister invisus sp. nov., isolated from the human oral cavity. Int J Syst Evol Microbiol 53, 1937-1940.

Eckburg, P. B., Bik, E. M., Bernstein, C. N., Purdom, E., Dethlefsen, L., Sargent, M., Gill, S. R., Nelson, K. E. \& Relman, D. A. (2005). Diversity of the human intestinal microbial flora. Science 308, 1635-1638.

Ezaki, T., Saidi, S. M., Liu, S. L., Hashimoto, Y., Yamamoto, H. \& Yabuuchi, E. (1990). Rapid procedure to determine the DNA base composition from small amounts of gram-positive bacteria. FEMS Microbiol Lett 55, 127-130.

Felsenstein, J. (1993). PHYLIP (phylogeny inference package), version 3.5c. Distributed by the author. Department of Genome Sciences, University of Washington, Seattle, USA.

Gill, S. R., Pop, M., Deboy, R. T., Eckburg, P. B., Turnbaugh, P. J., Samuel, B. S., Gordon, J. I., Relman, D. A., Fraser-Liggett, C. M. \&
Nelson, K. E. (2006). Metagenomic analysis of the human distal gut microbiome. Science 312, 1355-1359.

Holdeman, L. V., Cato, E. P. \& Moore, W. E. C. (1977). Anaerobe Laboratory Manual, 4th edn. Blacksburg, VA: Virginia Polytechnic Institute and State University.

Jumas-Bilak, E., Jean-Pierre, H., Carlier, J.-P., Teyssier, C., Bernard, K., Gay, B., Campos, J., Morio, F. \& Marchandin, H. (2005). Dialister micraerophilus sp. nov. and Dialister propionicifaciens sp. nov., isolated from human clinical samples. Int J Syst Evol Microbiol 55, 2471-2478.

Ley, R. E., Turnbaugh, P. J., Klein, S. \& Gordon, J. I. (2006). Microbial ecology: human gut microbes associated with obesity. Nature 444, 1022-1023.

Lipman, D. J. \& Pearson, W. R. (1985). Rapid and sensitive protein similarity searches. Science 227, 1435-1441.

Mai, V., Greenwald, B., Morris, J. G., Jr, Raufman, J. P. \& Stine, O. C. (2006). Effect of bowel preparation and colonoscopy on postprocedure intestinal microbiota composition. Gut 55, 1822-1823.

Moore, L. V. H. \& Moore, W. E. C. (1994). Oribaculum catoniae gen. nov., sp. nov.; Catonella morbi gen. nov., sp. nov.; Hallella seregens gen. nov., sp. nov.; Johnsonella ignava gen. nov., sp. nov.; and Dialister pneumosintes gen. nov., comb. nov., nom. rev., anaerobic gramnegative bacilli from the human gingival crevice. Int J Syst Bacteriol 44, 187-192.

Page, R. D. M. (1996). TreeView: an application to display phylogenetic trees on personal computers. Comput Appl Biosci 12, 357-358.

Rajilić-Stojanović, M., Smidt, H. \& de Vos, W. M. (2007). Diversity of the human gastrointestinal tract microbiota revisited. Environ Microbiol 9, 2125-2136.

Saitou, N. \& Nei, M. (1987). The neighbor-joining method: a new method for reconstructing phylogenetic trees. Mol Biol Evol 4, 406-425.

Sakamoto, M., Lan, P. T. N. \& Benno, Y. (2007). Barnesiella viscericola gen. nov., sp. nov., a novel member of the family Porphyromonadaceae isolated from chicken caecum. Int J Syst Evol Microbiol 57, 342346.

Sakon, H., Nagai, F., Morotomi, M. \& Tanaka, R. (2008). Sutterella parvirubra sp. nov. and Megamonas funiformis sp. nov., isolated from human faeces. Int J Syst Evol Microbiol 58, 970-975.

Tamura, K., Dudley, J., Nei, M. \& Kumar, S. (2007). MEGA4: molecular evolutionary genetics analysis (MEGA) software version 4.0. Mol Biol Evol 24, 1596-1599.

Thompson, J. D., Gibson, T. J., Plewniak, F., Jeanmougin, F. \& Higgins, D. G. (1997). The CLUSTAL_X windows interface: flexible strategies for multiple sequence alignment aided by quality analysis tools. Nucleic Acids Res 25, 4876-4882.

Willems, A. \& Collins, M. D. (1995). Phylogenetic placement of Dialister pneumosintes (formerly Bacteroides pneumosintes) within the Sporomusa subbranch of the Clostridium subphylum of the grampositive bacteria. Int J Syst Bacteriol 45, 403-405. 